in vivo $36: 49-56(2022)$

doi:10.21873/invivo.12675

\title{
Thoracic Neuroblastoma: A Novel Surgical Model for the Study of Extra-adrenal Neuroblastoma
}

\author{
CHRISTA N. GRANT ${ }^{1}$, CARSON A. WILLS ${ }^{2}$, XIAOMING LIU ${ }^{2}$, \\ VLADIMIR S. SPIEGELMAN ${ }^{2}$ and HONG-GANG WANG ${ }^{2}$ \\ ${ }^{1}$ Department of Pediatric Surgery, Milton S. Hershey Medical Center, Hershey, PA, U.S.A.; \\ ${ }^{2}$ Department of Pediatrics, Division of Pediatric Hematology and Oncology, \\ Penn State College of Medicine, Hershey, PA, U.S.A.
}

\begin{abstract}
Background/Aim: Neuroblastoma is clinically and molecularly heterogeneous, with poor outcomes despite multimodal treatment strategies. The primary tumor site is an independent predictor of survival; adrenal tumors have the worst outcomes, while posterior mediastinum tumors carry a more favorable prognosis. Materials and Methods: To elucidate the role of the primary tumor microenvironment in mediating survival outcomes, we developed a mouse model for the study of extra-adrenal neuroblastoma by injecting luciferase-tagged cells into either the subpleural space of the posterior chest or the adrenal gland. Results: Solid tumors developed in the thoracic cavity at the same rate and efficiency as the adrenal as early as one week postsurgery. The survival rate following surgery was equivalent, though the physiological tolerance for large tumors was lower in the thoracic group. Conclusion: This novel mouse model of survivable extra-adrenal neuroblastoma will enable future investigations of the distinct tumor microenvironments between the adrenal gland and posterior mediastinum.
\end{abstract}

Neuroblastoma is the most common pediatric extracranial solid tumor, accounting for $8-10 \%$ of all childhood tumors and approximately $15 \%$ of all pediatric cancer-associated deaths (1). Tumors are both clinically and molecularly heterogenous, with widely varying outcomes that are

This article is freely accessible online.

Correspondence to: Christa N. Grant, Department of Pediatric Surgery, H113 Milton S. Hershey Medical Center, 500 University Drive, P.O. Box 850, Hershey, PA 17033, U.S.A. E-mail: christagrantmd@gmail.com

Key Words: Neuroblastoma, adrenal, extra-adrenal, thoracic, posterior mediastinum, mouse model, orthotopic model. dependent on patient age, tumor stage, pathologic and biologic markers, and tumor location (2). While the overall survival rate for fully resected, localized tumors with favorable histology is over $85 \%$, the survival rate drops to just $34 \%$ for stage IV tumors despite multimodal therapy (3). The most common genetic aberration associated with poor outcomes in neuroblastoma is amplification of the $M Y C N$ oncogene, found in $16-25 \%$ of primary tumors (4). Primary tumor site has also been identified as an independent predictor of survival in all risk groups, regardless of $M Y C N$ amplification status: adrenal tumors have the worst outcomes, while thoracic and cervical tumors, which account for approximately $15 \%$ of all primary neuroblastomas, generally carry a more favorable prognosis $(5,6)$. In 2014 , the largest comprehensive analysis of primary tumor site in neuroblastoma studied 8,369 children from the International Neuroblastoma Risk Group (INRG) database (1990-2002) and found that patients with primary adrenal tumors had higher rates of bone marrow, bone, and liver metastasis, as well as elevated ferritin, elevated LDH, and increased rates of segmental chromosomal aberrations compared to patients with primary thoracic neuroblastoma (5). Despite the welldocumented survival advantage of extra-adrenal tumors in clinical reports, few researchers have investigated primary tumor site as an independent variable for understanding tumor behavior. Elucidating the molecular mechanisms underlying this difference in survival rate between primary tumor sites is critically important for identifying novel biological markers and therapeutic targets, particularly for patients with high-risk $M Y C N$ non-amplified tumors.

Genetically engineered mouse models (GEMMs) and surgical models are the standard techniques utilized to study neuroblastoma in vivo $(7,8)$. Recent advances in ultrasoundguided adrenal injections reliably yield patient-derived tumor xenografts (9). While several GEMMS have extra-adrenal manifestations, none have reliably yielded thoracic neuroblastoma for study, and there are no surgical models of 
neuroblastoma published that specifically evaluate extraadrenal tumor sites, limiting the ability to study the molecular differences and therapeutic options between adrenal and thoracic primary tumors. In this study, we set out to develop a novel, survivable mouse model for the study of extra-adrenal neuroblastoma.

\section{Materials and Methods}

Cell culture. The mouse neuroblastoma cell line 9464D was a gift from Dr. Paul Sondel's lab (University of Wisconsin, Madison). Cells were cultured in DMEM (Dulbecco's Modified Eagle's Medium) with $4.5 \mathrm{~g} / \mathrm{l}$ glucose, L-glutamine, and sodium pyruvate supplemented with $10 \%(\mathrm{v} / \mathrm{v})$ heat-inactivated FBS (Sigma-Aldrich F2442) and $1 \%$ antibiotic-antimycotic solution (Corning MT300004CI) at $37^{\circ} \mathrm{C}$ and $5 \% \mathrm{CO}_{2}$ in a humidified incubator. Luciferase reporter cells were transduced with a pCDH1-SV40Luc2 construct and selected with hygromycin $(200 \mu \mathrm{g} / \mathrm{ml})$.

Extra-adrenal tumor mouse model. Neuroblastoma cells were suspended in a 50:50 mixture of PBS and Matrigel basement membrane matrix (Fisher scientific) for a total injected volume of $10 \mu \mathrm{l}$. Eight- to 12 -week-old mice were anesthetized with inhaled isoflurane. The fur over the left hemithorax was shaved with a clipper. Antibiotic eye lubricant was applied. The mouse was laid on its right ride on a warmer and the left chest skin cleaned with alcohol and betadine swabs. All subsequent steps were performed sterilely, and the entire procedure took place in a protective hood. A $1 \mathrm{~cm}$ skin incision was made above the left $7^{\text {th }}$ rib. The chest wall muscles were retracted, the intercostal muscle was opened, and the chest entered. Once the chest was opened, the left lung deflated, and the inner chest wall was easily visualized. The cells were injected just beneath the pleura between the $5^{\text {th }}$ and $6^{\text {th }}$ rib using a Hamilton syringe (Hamilton Company, Reno, NA, USA). The needle was withdrawn and the ribs were reapproximated with a single absorbable suture through the intercostal muscles. A $1 \mathrm{ml}$ syringe containing $0.3 \mathrm{ml}$ saline with an attached 28 Gauge needle was placed through the muscle and into the chest while aspirating the atmospheric air from the chest, allowing the lung to fully re-expand. The chest wall muscles and skin were closed with a running absorbable suture. Mice were allowed to awaken within a warmed oxygen chamber. Mice were housed individually and fed standard chow and water. Injectable normal saline and ketoprofen $5 \mathrm{mg} / \mathrm{kg}$ were injected subcutaneously (SC) just prior to surgery then daily for three days. Weights were checked weekly and compared to preoperative weights. Mice were sacrificed at 4 weeks, or sooner if they lost more than $10 \%$ body weight or reached humane endpoints. The model was developed using 9464D mouse neuroblastoma cells injected into C57BL/6J mice (Jackson laboratories), and later replicated successfully with implantation of multiple human-derived neuroblastoma cell lines into NRG mice (Jackson laboratories). This study was approved by the Penn State College of Medicine institutional animal care and use committee (IACUC Protocol \#202001564).

Adrenal tumor mouse model. Adrenal injections were performed via left subcostal laparotomy incision as previously described and shown in Figure $1(10,11)$.

Luciferase tumor imaging. Primary tumor growth and metastasis was evaluated weekly using IVIS Lumina III in vivo bioluminescent imaging (IVIS). $30 \mathrm{mg} / \mathrm{ml}$ D-luciferin was dissolved in PBS; mice were anesthetized with $2.5 \%$ isoflurane (IsoSol Isoflurane, USP, VEDCO) and injected subcutaneously at a dose of $5 \mu \mathrm{l} / \mathrm{g}$ body weight $5 \mathrm{~m}$ prior to imaging. Photon flux was calculated using region of interest (ROI) measurements around the primary tumor site.

Histologic analysis. Primary tumors were collected, fixed in formalin, paraffin embedded, and stained with hematoxylin and eosin (H\&E) for histologic analysis.

Ultrasound-guided imaging. Tumors were imaged just prior to the end point and tumor harvest. The Visualsonics Vevo 2100 ultrasound imaging system (Visualsonics, Toronto, Canada) was used to measure tumor location and anatomic relationships.

\section{Results}

Development of a novel survival mouse model of extraadrenal neuroblastoma. In order to establish a novel mouse model of extra-adrenal neuroblastoma, we first used a wellestablished subcostal laparotomy protocol to seed luciferaselabeled 9464D neuroblastoma tumor cells into the left adrenal gland of immunocompetent mice (Figure 1A-E) (11, 12). This allowed us to both validate our tumor cell line and the surgical technique. Mice with primary adrenal tumors seeded using this approach served as controls throughout the development of our model of extra-adrenal neuroblastoma.

Thoracic and cervical neuroblastomas account for approximately $15 \%$ of all primary tumors, but have been demonstrated to have a favorable prognosis compared to primary adrenal tumors (6). To establish a mouse model of primary thoracic neuroblastoma, we performed thoracotomies by making $1 \mathrm{~cm}$ incisions above the left seventh rib of anesthetized, immunocompetent mice, and retracting the chest wall and intercostal muscles (Figure 2A-C). Once the chest wall was open, the lung deflated, permitting visualization of the inner chest wall cavity, the vertebral bodies, and the articulating ribs, as well as the parietal pleura, which is a thin epithelial layer that separates the chest cavity from the chest wall (Figure 2D). A Hamilton syringe was used to inject 200,000 luciferase-labeled 9464D tumor cells suspended in a 1:1 mixture of Matrigel basement membrane matrix into the subpleural space between the left fifth and sixth rib of immunocompetent mice, approximating the location of primary human neuroblastoma growth along the sympathetic trunk in the paravertebral region (Figure 2E). The ribs were then re-approximated with a suture through the intercostal muscles, allowing the chest cavity to become air tight. To prevent pneumothorax and allow the left lung to re-inflate, a 28 Gauge needle was used to aspirate all remaining atmospheric air from the chest cavity (Figure 2F). Mice were then allowed to spontaneously awaken from anesthesia and monitored for post-surgical complications (Figure 2G). Titration experiments were conducted, and 200,000 9464D cells was the optimal number of cells that allowed reliable 

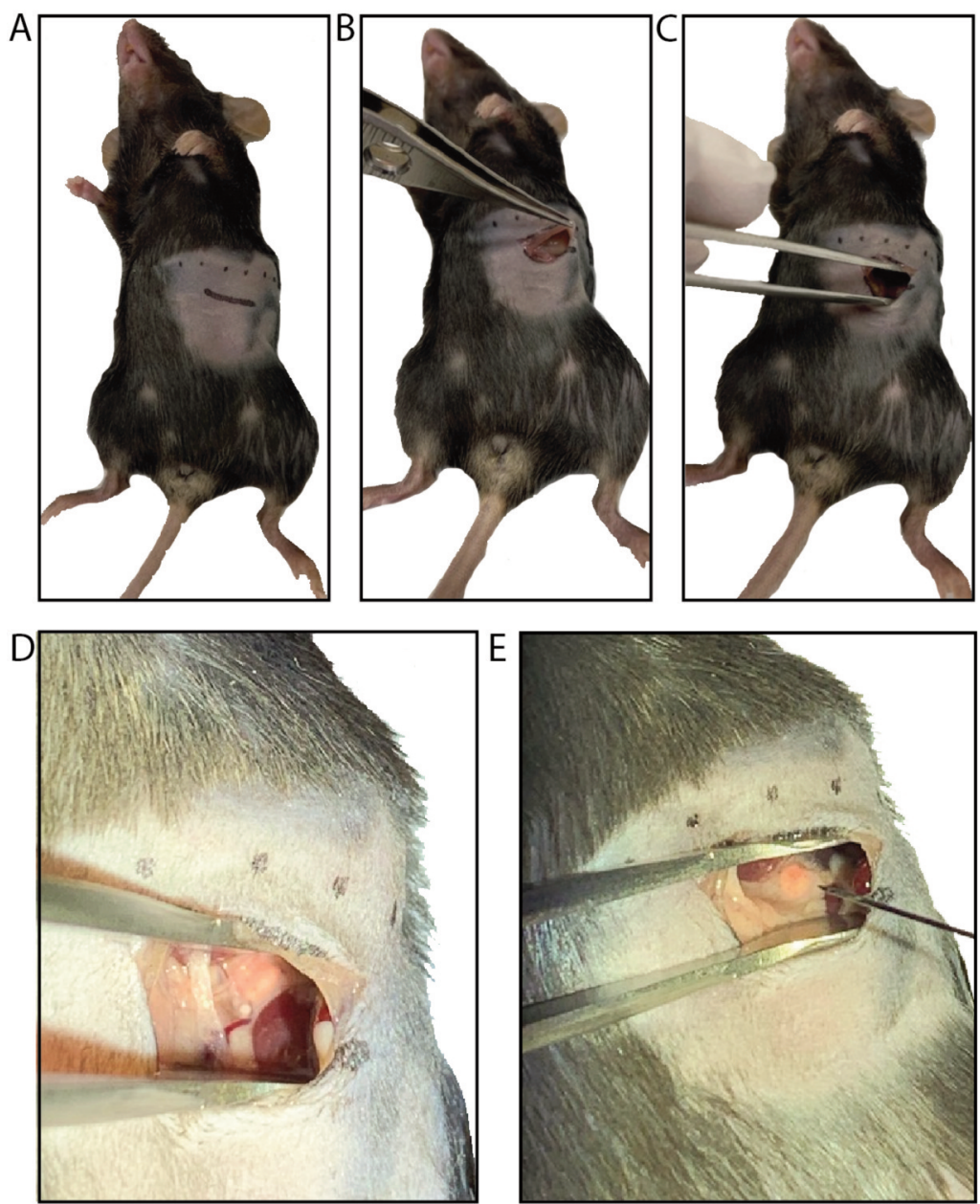

Figure 1. Surgical technique to establish primary adrenal neuroblastoma tumors. (A) The mouse was anesthetized, shaved, and the subcostal space was using anatomical landmarks. (B) A subcostal incision was performed. (C-D) The left adrenal gland was visualized. (E) Neuroblastoma cells were injected into the left adrenal gland.

tumor yield and IVIS detection. Notably, the model has since been reproduced in our lab using multiple other neuroblastoma cell lines, some of which require higher numbers of cells for optimal tumor yield.

Survival and post-surgical outcomes. In order to determine the viability and reproducibility of our extra-adrenal neuroblastoma model, we first compared survival outcomes between the adrenal and thoracic groups intraoperatively, during the post-operative period, and long-term. During the first set of experiments using 9464D-tumor bearing immunocompetent mice, $100 \%$ of mice that underwent subcostal laparotomy survived surgery and recovered without complications. All mice in this group developed viable tumors in the abdominal cavity. By post-operative week 4 the tumors became large and necrotic (Figure 3B), likely due to a decreased blood supply, and mice were humanely sacrificed.
In mice that underwent thoracotomy with subpleural injection of neuroblastoma cells, $5 \%$ of mice succumbed to anesthesia or surgery, for a surgical survival rate of $95 \%$. In mice that survived surgery, $100 \%$ recovered and survived in the immediate postoperative period. All animals that underwent thoracotomy developed viable tumors in the intrathoracic region, some of which grew so large that they became transthoracic with a large dorsal component (Figure 3C-D). All tumors were distinct from the lung and grew along the chest wall pleura where cells were implanted, suggesting that the injection technique was successful with no tumor spillage. Given that large intrathoracic tumors grew in all mice, leading to decreased appetite and signs of respiratory distress, the week 3 timepoint was chosen as the humane endpoint for mice in the thoracic neuroblastoma group.

Overall, intraoperative and postoperative survival rates between subcostal laparotomies and thoracotomies were 

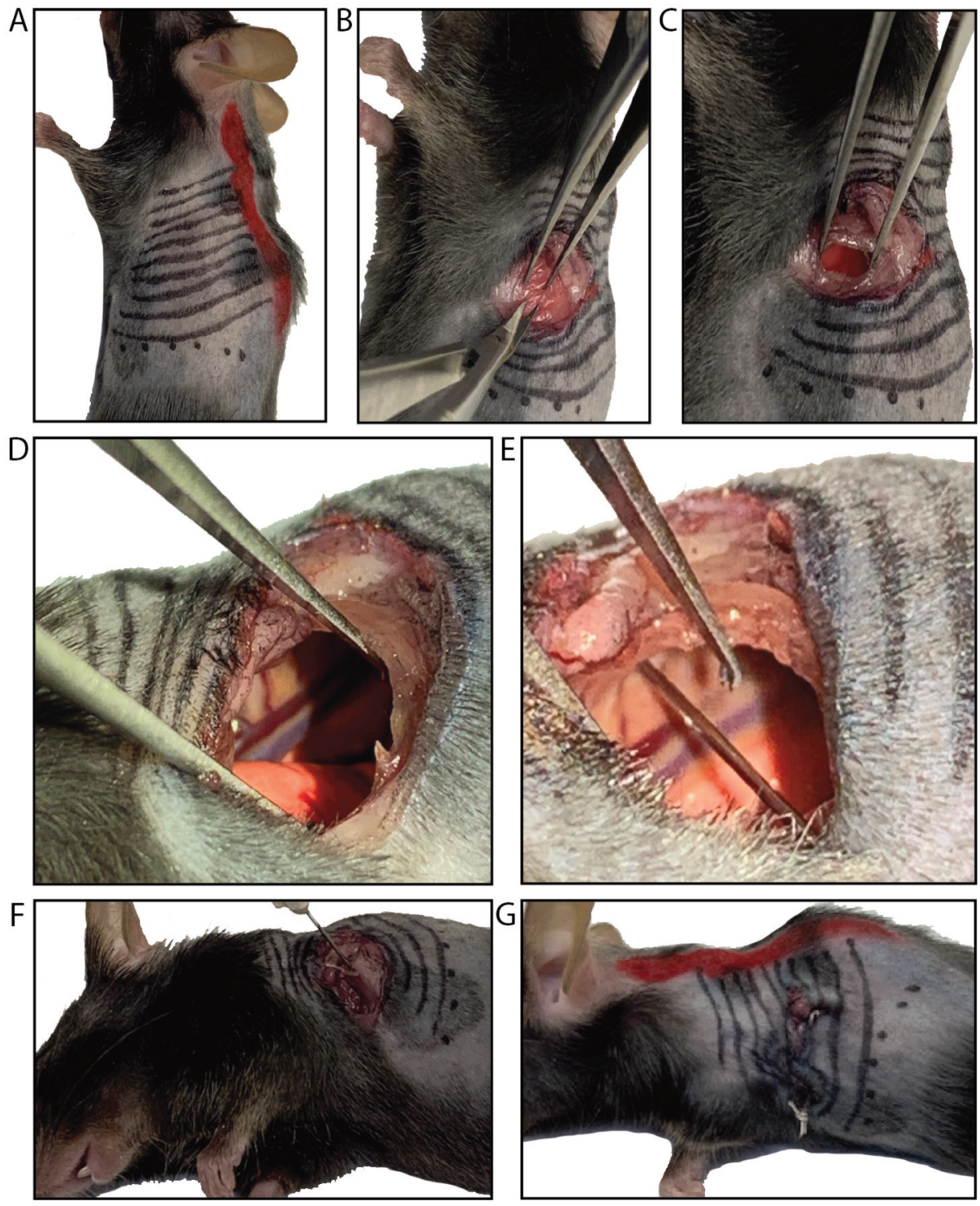

Figure 2. Surgical technique to establish extra-adrenal primary neuroblastoma tumors. (A) The mouse was anesthetized, shaved, and intervertebral spaces were visualized using anatomical landmarks. (B) A $1 \mathrm{~cm}$ skin incision was made above the left $7^{\text {th }}$ rib. (C) The chest wall muscles were retracted, the intercostal muscle was opened, and the chest entered. (D) Once the chest was opened, the left lung deflated, allowing visualization of the vertebral bodies and articulating ribs. (E) Neuroblastoma cells were injected just beneath the pleura between the $5^{\text {th }}$ and $6^{\text {th }}$ rib. $(F)$ The rib was reapproximated with a single suture and air was aspirated after closing the muscle layer, allowing the lung to re-expand. $(G)$ The incision was sutured, and the mouse monitored for complications post-surgery.

equivalent and reproducible. Mice that underwent laparotomy and adrenal tumor cell injections survived an average of one week longer than mice that underwent thoracotomy and received subpleural injections, likely due to the mass effect of intrathoracic tumors on the lung and heart (Figure 3A) within the fixed thoracic cavity; in contrast, the abdomen and retroperitoneum expanded to accommodate the very large adrenal tumors. Notably, across multiple experimental replicates, gross metastatic lesions were only visible in the lungs and liver of mice in the adrenal primary tumor group (Figure $3 \mathrm{G}-\mathrm{H})$. This phenotype may reflect the longer duration of survival in this experimental model, or it could reflect intrinsic differences between the primary tumor microenvironment in these two models leading to suppression of metastatic tumor progression in thoracic tumors. This finding is notable given the human data suggesting that outcomes are worse for patients with primary adrenal rather than thoracic tumors and warrants further experimental investigation. 

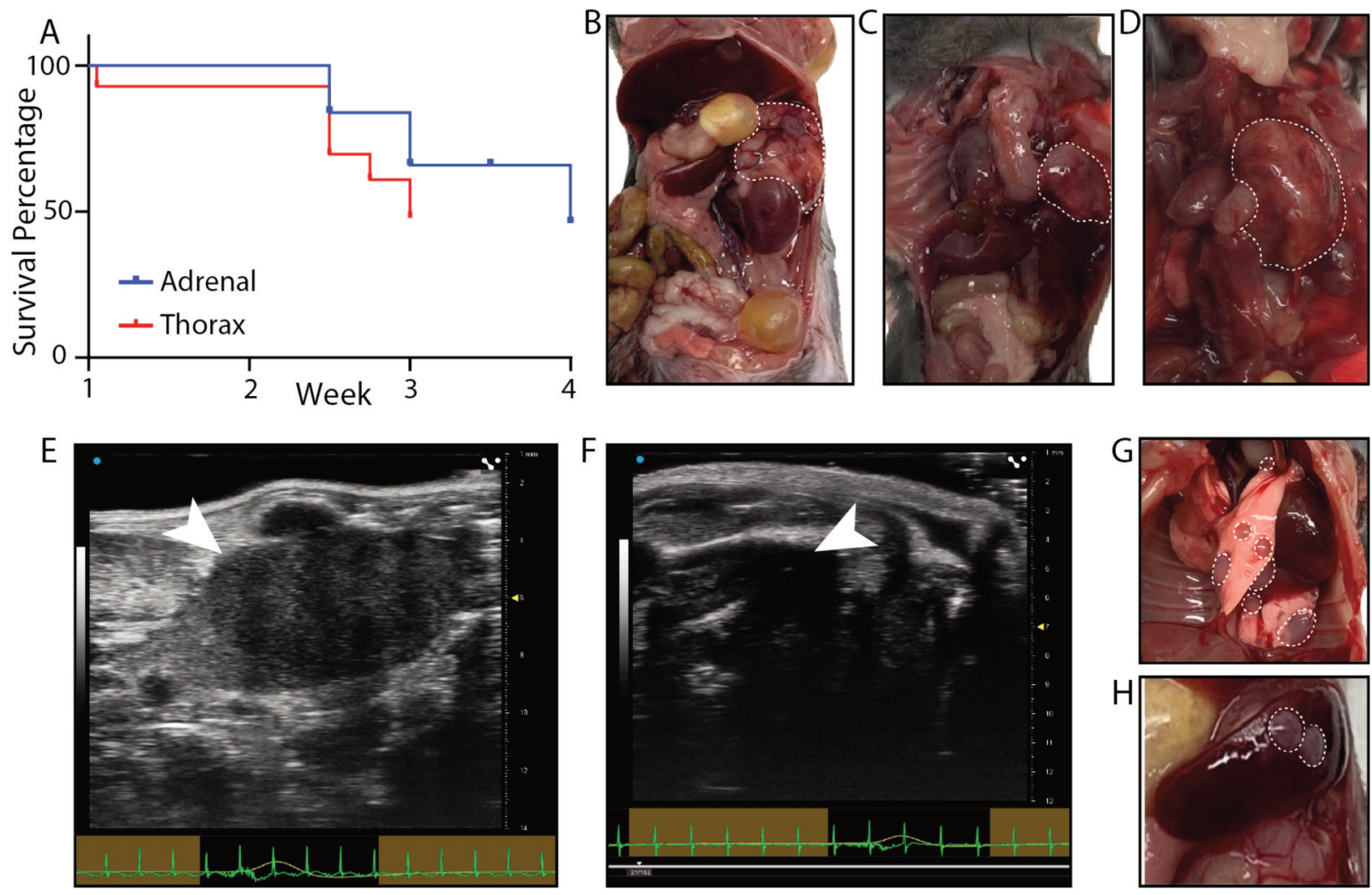

Figure 3. Primary tumor growth and metastasis in vivo. (A) Combined survival proportion for all experiments with 9464D neuroblastoma cells combined. Adrenal tumors $n=22$, thorax tumors $n=15$. (B) Representative image of primary adrenal tumor (outlined) at week 4. (C-D) Representative image of primary thorax tumor (outlined) at week 4. (E) Ultrasound-guided image of primary adrenal tumor (white arrowhead) at week $4 .(F)$ Ultrasound-guided image of thoracic tumor (white arrowhead) at week 4. (G) Primary adrenal tumor metastatic (outlined) to the lung. (H) Primary adrenal tumor metastatic (outlined) to the liver.

Characterization of primary adrenal and thoracic tumors. In order to determine whether our novel intra-thoracic mouse model produced viable neuroblastoma tumors comparable to established orthotopic models of adrenal neuroblastoma, we utilized ultrasound and in vivo bioluminescent imaging to quantify primary tumor growth over time, as well as histologic analysis to analyze primary tumors ex vivo.

In vivo ultrasound-guided imaging of mice in the control group demonstrated viable tumors within the abdominal cavity at the apex of the left kidney (Figure 3E). Similarly, ultrasound of mice in the thoracotomy group revealed tumors within the thoracic cavity that were distinct from the lung, confirming successful implantation of and growth of tumor cells (Figure 3F). To confirm successful implantation of tumor cells, as well as track and quantify tumor growth over time, in vivo bioluminescent imaging of luciferase-labeled primary tumor cells was utilized. Imaging demonstrated a bioluminescent signal confined to either the thoracic or abdominal region, which increased in intensity each week, confirming tumor viability and growth (Figure 4A-C).
Notably, bioluminescent signal was primarily confined to the region of tumor cell implantation, suggesting little metastatic growth, in concordance with ex vivo gross tissue analysis.

Finally, to characterize tumors from both the thoracic cavity and adrenal glands, primary tumors were isolated at the conclusion of the experiment, fixed, and stained with hematoxylin and eosin. All samples demonstrated the characteristic small round blue cells in a background of neural tissue and neuropil, confirming that they were neuroblastic in origin (Figure 5A-D). Notably, no significant differences in cell composition were noted on histologic analysis, suggesting that the microenvironment in the thoracic cavity is conducive to neuroblastoma cell growth in this model.

\section{Discussion}

In this study we demonstrated a novel and highly-reproducible surgical technique to establish extra-adrenal neuroblastoma in an immunocompetent mouse model, with the goal of 

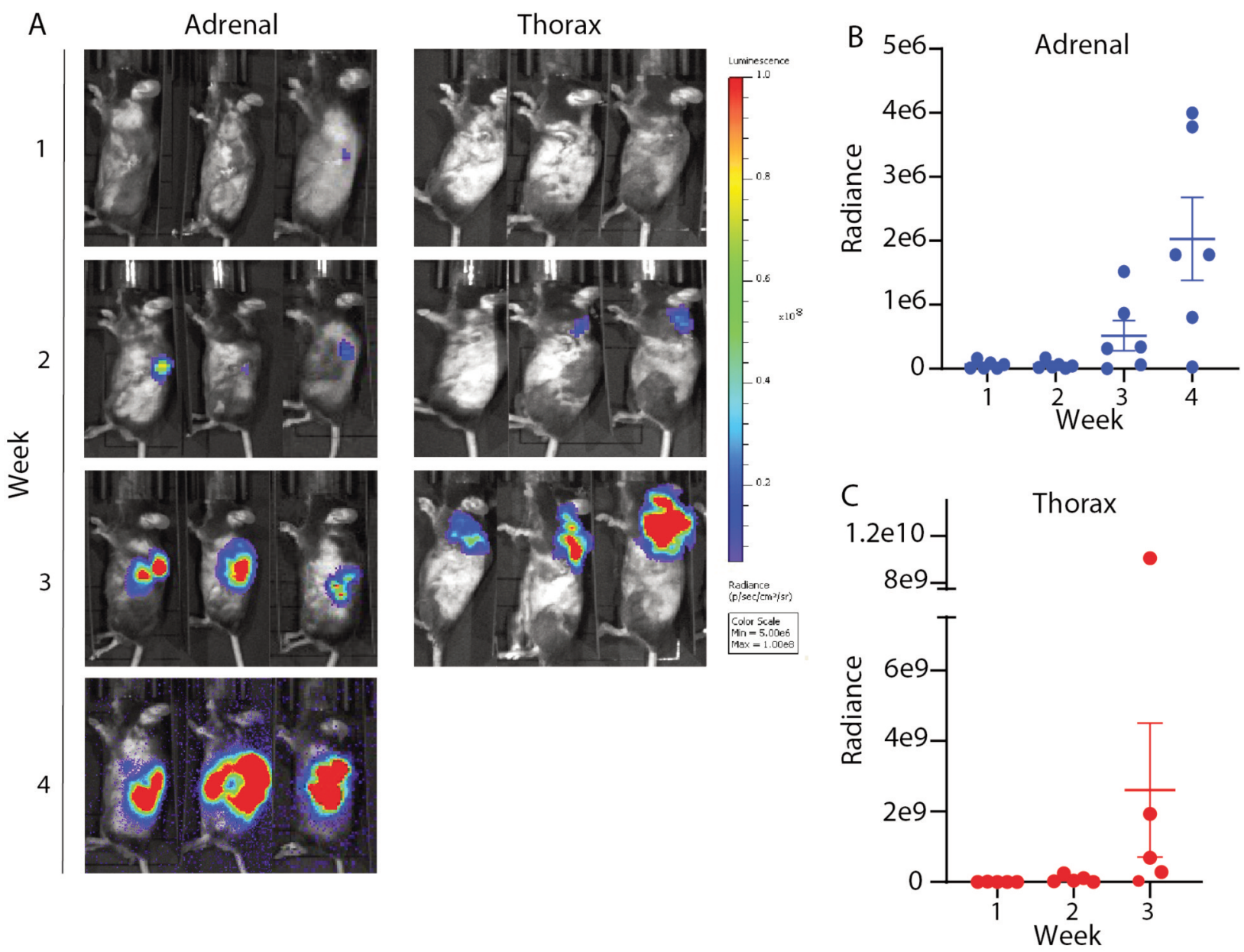

Figure 4. Quantification of primary tumor luciferase expression. (A) Representative bioluminescent images of mice with adrenal (left) and thoracic (right) primary tumors at indicated time points. (B) Quantification of adrenal primary tumor region of interest (ROI) radiance at indicated time points, $n=6$. (C) Quantification of thoracic primary tumor region of interest (ROI) radiance at indicated time points, $n=5$.

investigating the role of primary tumor site in neuroblastoma tumor progression. To our knowledge, this is the first surgical model of neuroblastoma outside the adrenal gland in a location analogous to human disease. We demonstrated that our surgical technique is not only reproducible, but also yields tumors that are phenotypically similar to tumors grown in the adrenal gland. The survival advantage of patients with extra-adrenal compared to adrenal neuroblastoma is well-documented in the literature, but the underlying molecular mechanisms that regulate these site-specific differences remain poorly characterized. This novel orthotopic model of survivable extrathoracic neuroblastoma will enable elucidation of the biologic and microenvironmental differences between tumor subtypes that confer this survival advantage, in order to develop more effective and targeted therapeutic options for high-risk patients.

Several surgical and genetically engineered mouse models (GEMMs) of neuroblastoma exist (13). Metastatic invasion models have the drawback of poor reproducibility and dependence on primary tumor characteristics. Transgenic animal models tend to better recapitulate human tumor behavior and allow for the study of specific mutations, but genetic alteration is necessary to induce the model. Syngeneic models are highly reproducible after carcinogen administration, but poorly recapitulate human tumor behavior. Humanized animal models have little translatability to solid tumor biology and are primarily used for hematopoietic reconstitution, microbial infection, and vaccine development (13). Orthotopic mouse models are superior to subcutaneous models in recapitulating the tumor microenvironment and human disease, but are primarily limited to the adrenal gland in neuroblastoma, which does not fully represent the spectrum of human disease. While a few orthotopic mouse models of neuroblastoma spontaneously developed tumors in the thorax in addition to 

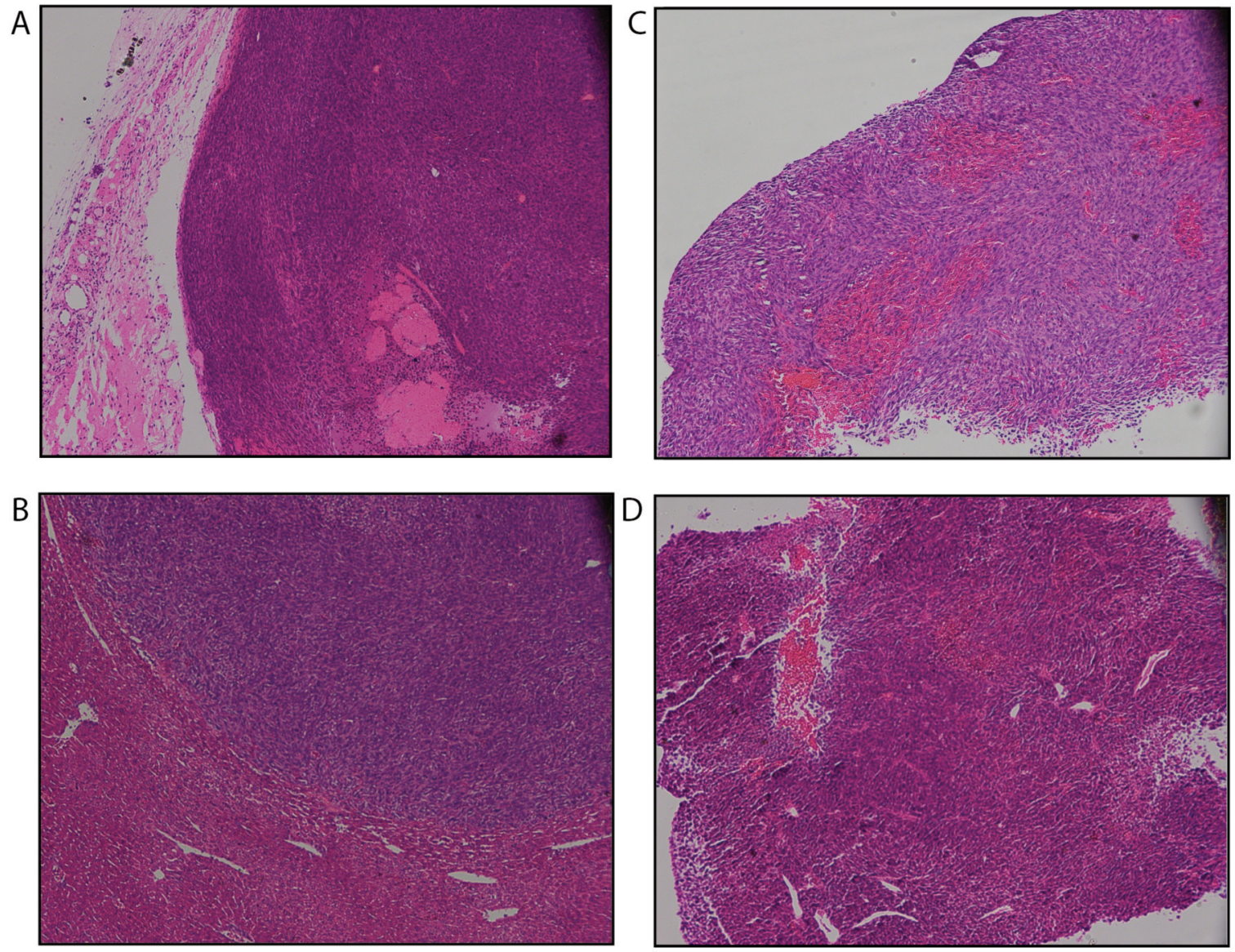

Figure 5. Representative histology of primary tumors ex vivo. (A-B) Representative $H \& E$ section of primary adrenal tumors. (C-D) Representative $H \& E$ section of primary thoracic tumors.

other body cavities, there has not been a specific thoracic neuroblastoma model thus far.

Our model makes it possible, for the first time, to investigate the distinct tumor microenvironments of both the adrenal gland and the posterior mediastinum, which represent the two most common primary sites of neuroblastoma, but carry vastly different survival outcomes for patients. This is the first surgical model to yield posterior mediastinal tumors and maintain mouse survival for downstream experiments, which will enable further characterization of the biological differences between tumors grown in distinct tumor microenvironments. Advantages of this technique include its reproducibility and ability to recapitulate human disease, as well as the short period of time from incision to closure, which minimizes the risks to mice associated with prolonged exposure to anesthesia.

Limitations of the technique include a lack of feasibility to implant tumor cells directly into the paraspinal ganglia, which would more accurately represent human disease. However, we believe that tumors grown in the subpleural space of the posterior mediastinum is the closest feasible recapitulation of thoracic neuroblastoma, and will still yield valuable information about the tumor microenvironment of the thoracic cavity. In addition, tumors occasionally grow through the chest wall rather than inward toward the chest cavity after implantation beneath the pleural lining, which can lead to large chest wall tumors. This finding was eliminated after the first two experimental trials when the needle used for injection was changed to a small Hamilton syringe and care taken not to inject deeper than the subpleural space. Rapid growth of intra-thoracic tumors leads to compression of the cardiovascular system, and mice with these tumors may reach a humane endpoint earlier than mice with primary adrenal tumors, so titration experiments to determine the optimal number of cells for implantation are recommended for each cell line. For instance, lower numbers of tumor cells may yield smaller tumors, and if physiologic tolerance of smaller tumors improves, longer endpoints may be attained in order to determine additional information, such as late metastasis. In our model, mice with thoracic tumors 
that survived to six weeks did not demonstrate any metastasis or additional clear phenotype differences. Finally, this surgical model has a steep learning curve, which can limit the generalizability of this approach.

Using our innovative mouse model, future studies will be able to investigate the role that the distinct tumor microenvironments play in the survival differences between these two neuroblastoma tumor locations. Identification of the underlying mechanisms regulating tumor behavior in distinct physiologic locations will help elucidate the differences in clinical outcomes between groups and will open innovative new avenues for therapeutic targeting.

\section{Conflicts of Interest}

The Authors declare no conflicts of interest.

\section{Authors' Contributions}

C.N.G conceived and planned this study. C.N.G., C.A.W, and X.L. designed and performed the experiments. C.N.G. and C.A.W. analyzed the data, prepared the figures, and wrote the paper with feedback from all Authors. V.S.S. provided experimental reagents, and helped with data interpretation. C.N.G. and H.G.W. planned, interpreted, and supervised this study.

\section{Acknowledgements}

The Authors acknowledge Jennifer Wilson and Longgui Chen for their technical assistance and animal husbandry. This work was supported by the APSA foundation and Four Diamonds Fund of Penn State College of Medicine.

\section{References}

1 Colon NC and Chung DH: Neuroblastoma. Adv Pediatr 58(1): 297311, 2011. PMID: 21736987. DOI: 10.1016/j.yapd.2011.03.011

2 Howlander N, Noone A, Krapcho M, Miller D, Brest A, Yu M, Ruhl J, Tatalovich Z, Mariotto A, Lewis D, Chen H, Feuer E and Cronin K: SEER Cancer Statistics Review, 1975-2016, National Cancer Institute. Available at: https://seer.cancer.gov/archive/csr/1975_2016/ [Last accessed on October 11, 2021]

3 Escobar MA, Grosfeld JL, Powell RL, West KW, Scherer LR 3rd, Fallon RJ and Rescorla FJ: Long-term outcomes in patients with stage IV neuroblastoma. J Pediatr Surg 41(2): 377-381, 2006. PMID: 16481255 . DOI: 10.1016/j.jpedsurg.2005.11.032

4 Ambros PF, Ambros IM, Brodeur GM, Haber M, Khan J, Nakagawara A, Schleiermacher G, Speleman F, Spitz R, London WB, Cohn SL, Pearson AD and Maris JM: International consensus for neuroblastoma molecular diagnostics: report from the International Neuroblastoma Risk Group (INRG) Biology Committee. Br J Cancer 100(9): 1471-1482, 2009. PMID: 19401703. DOI: $10.1038 /$ sj.bjc.6605014
5 Vo KT, Matthay KK, Neuhaus J, London WB, Hero B, Ambros PF, Nakagawara A, Miniati D, Wheeler K, Pearson AD, Cohn SL and DuBois SG: Clinical, biologic, and prognostic differences on the basis of primary tumor site in neuroblastoma: a report from the international neuroblastoma risk group project. J Clin Oncol 32(28): 3169-3176, 2014. PMID: 25154816. DOI: 10.1200/JCO.2014.56.1621

6 Parikh D, Short M, Eshmawy M and Brown R: Surgical outcome analysis of paediatric thoracic and cervical neuroblastoma. Eur J Cardiothorac Surg 41(3): 630-634, 2012. PMID: 22011775. DOI: 10.1093/ejcts/ezr005

7 Weiss WA, Aldape K, Mohapatra G, Feuerstein BG and Bishop JM: Targeted expression of MYCN causes neuroblastoma in transgenic mice. EMBO J 16(11): 2985-2995, 1997. PMID: 9214616. DOI: 10.1093/emboj/16.11.2985

8 Althoff K, Beckers A, Bell E, Nortmeyer M, Thor T, Sprüssel A, Lindner S, De Preter K, Florin A, Heukamp LC, Klein-Hitpass L, Astrahantseff K, Kumps C, Speleman F, Eggert A, Westermann F, Schramm A and Schulte JH: A Cre-conditional MYCN-driven neuroblastoma mouse model as an improved tool for preclinical studies. Oncogene 34(26): 3357-3368, 2015. PMID: 25174395. DOI: 10.1038/onc.2014.269

9 Thomas TT, Chukkapalli S, Van Noord RA, Krook M, Hoenerhoff MJ, Dillman JR, Lawlor ER, Opipari VP and Newman EA: Utilization of ultrasound guided tissue-directed cellular implantation for the establishment of biologically relevant metastatic tumor xenografts. J Vis Exp (135): 57558, 2018. PMID: 29889191. DOI: 10.3791/57558

10 Daudigeos-Dubus E, LE Dret L, Rouffiac V, Bawa O, Leguerney I, Opolon P, Vassal G and Geoerger B: Establishment and characterization of new orthotopic and metastatic neuroblastoma models. In Vivo 28(4): 425-434, 2014. PMID: 24982206.

11 Cardoso CC, Bornstein SR and Hornsby PJ: Optimizing orthotopic cell transplantation in the mouse adrenal gland. Cell Transplant 19(5): 565-572, 2010. PMID: 20525431. DOI: 10.3727/096368910X509077

12 Marques da Costa ME, Daudigeos-Dubus E, Gomez-Brouchet A, Bawa O, Rouffiac V, Serra M, Scotlandi K, Santos C, Geoerger B and Gaspar N: Establishment and characterization of in vivo orthotopic bioluminescent xenograft models from human osteosarcoma cell lines in Swiss nude and NSG mice. Cancer Med 7(3): 665-676, 2018. PMID: 29473324. DOI: $10.1002 / \mathrm{cam} 4.1346$

13 Seitz G, Armeanu-Ebinger S, Warmann S and Fuchs J: Animal models of extracranial pediatric solid tumors. Oncol Lett 4(5): 859-864, 2012. PMID: 23162611. DOI: 10.3892/ol.2012.852

Received August 22, 2021

Revised September 28, 2021

Accepted October 11, 2021 